Port Acadie

Revue interdisciplinaire en études acadiennes

An Interdisciplinary Review in Acadian Studies

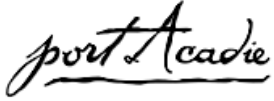

\title{
Une tentative de réhabilitation du patrimoine théâtral acadien : l'édition critique de Subercase ou les Dernières années de la domination française en Acadie d'Alexandre Braud (1902, 1936)
}

\section{Pierre Gérin}

Numéro 20-21, automne 2011, printemps 2012

L'édition critique et le développement du patrimoine littéraire en Acadie et dans les petites littératures

URI : https://id.erudit.org/iderudit/1010327ar

DOI : https://doi.org/10.7202/1010327ar

Aller au sommaire du numéro

Éditeur(s)

Université Sainte-Anne

ISSN

1498-7651 (imprimé)

1916-7334 (numérique)

Découvrir la revue

Citer cet article

Gérin, P. (2011). Une tentative de réhabilitation du patrimoine théâtral acadien : l'édition critique de Subercase ou les Dernières années de la domination française en Acadie d'Alexandre Braud $(1902,1936)$. Port Acadie, (20-21), 111-122. https://doi.org/10.7202/1010327ar
Résumé de l'article

À l'exclusion du Théâtre de Neptune de Marc Lescarbot (1609) et des Acadiens à Philadelphie de Pascal Poirier (1875), c'est sur les scènes des collèges que débute le théâtre acadien dont le chef-d'oeuvre est assurément Subercase Drame historique en trois actes, d'Alexandre Braud (1902). À cette époque, le théâtre collégial prend un grand essor au Canada français où il se distingue dans le genre dramatique. Cette oeuvre, louée par la critique et publiée en feuilleton dans Le Moniteur acadien, la même année, a une histoire mouvementée. Ayant perdu le manuscrit original, l'auteur réécrit partiellement, à Québec où il réside, la pièce qui est jouée sur une scène paroissiale de cette ville, en 1936, et qui appartient donc aussi au théâtre québécois. Après un long purgatoire, elle ressort de l'oubli : elle est présentée, citée et commentée dans des travaux d'histoire littéraire acadienne et dans des thèses. Pourtant, un obstacle à sa lecture et à sa diffusion réside dans son inaccessibilité. L'édition critique de ce drame est l'occasion de mettre celui-ci à la disposition du public lecteur dans la communauté acadienne et ailleurs dans la francophonie. Cependant, des choix éditoriaux s'imposent quant à l'établissement du texte, à la notation des variantes et à la constitution des appendices. Cette édition vise à faire connaître ou redécouvrir une forme d'art dramatique, certes tombée en désuétude, mais qui ressortit aux patrimoines culturels acadien, québécois et canadien-français.
Ce document est protégé par la loi sur le droit d'auteur. L'utilisation des services d’Érudit (y compris la reproduction) est assujettie à sa politique d'utilisation que vous pouvez consulter en ligne.

https://apropos.erudit.org/fr/usagers/politique-dutilisation/ 


\title{
Une tentative de réhabilitation du patrimoine théâtral acadien : l'édition critique de Subercase ou les Dernières années de la domination française en Acadie d'Alexandre Braud $(1902,1936)$
}

\author{
Pierre Gérin \\ Université de Moncton
}

\begin{abstract}
Résumé
À l'exclusion du Théâtre de Neptune de Marc Lescarbot (1609) et des Acadiens à Philadelphie de Pascal Poirier (1875), c'est sur les scènes des collèges que débute le théâtre acadien dont le chef-d'œuvre est assurément Subercase - Drame historique en trois actes, d'Alexandre Braud (1902). À cette époque, le théâtre collégial prend un grand essor au Canada français où il se distingue dans le genre dramatique. Cette œuvre, louée par la critique et publiée en feuilleton dans Le Moniteur acadien, la même année, a une histoire mouvementée. Ayant perdu le manuscrit original, l'auteur réécrit partiellement, à Québec où il réside, la pièce qui est jouée sur une scène paroissiale de cette ville, en 1936, et qui appartient donc aussi au théâtre québécois. Après un long purgatoire, elle ressort de l'oubli : elle est présentée, citée et commentée dans des travaux d'histoire littéraire acadienne et dans des thèses. Pourtant, un obstacle à sa lecture et à sa diffusion réside dans son inaccessibilité. L'édition critique de ce drame est l'occasion de mettre celui-ci à la disposition du public lecteur dans la communauté acadienne et ailleurs dans la francophonie. Cependant, des choix éditoriaux s'imposent quant à l'établissement du texte, à la notation des variantes et à la constitution des appendices. Cette édition vise à faire connaître ou redécouvrir une forme d'art dramatique, certes tombée en désuétude, mais qui ressortit aux patrimoines culturels acadien, québécois et canadien-français.
\end{abstract}

C'est sur les scènes des collèges que débute le théâtre acadien, si l'on ne tient compte ni du Théâtre de Neptune de Marc Lescarbot ${ }^{1}$ (1609) ni des deux pièces intégrales inédites de Pascal Poirier ${ }^{2}$ (1875). Assurément, le chef-d'œuvre du théâtre collégial acadien est Subercase - Drame historique en trois actes d'Alexandre Braud ${ }^{3}$, pièce jouée le 20 avril 1902,

1. Marc Lescarbot, "Le Théâtre de Neptune en la Nouvelle-France ", dans Les Muses de la Nouvelle-France, Paris, J. Millot, 1609, p. 17-27. Voir aussi : Bernard Émont, Les Muses de la Nouvelle-France de Marc Lescarbot, Paris, L’Harmattan, 2004, p. 131-145 (éd. crit.).

2. Pascal Poirier, "Les Acadiens à Philadelphie », 1875, CÉA, fonds 6.2-1A, 69 f.; P. Poirier, « Les Accordailles de Gabriel et d’Évangéline », [s.d., 1877], CÉA AnselmeChiasson, fonds 6.4-19, $22 \mathrm{f}$. Ces deux pièces ont fait l'objet d'une édition commune : P. Poirier, Les Acadiens à Philadelphie; suivi de Les Accordailles de Gabriel et d'Évangéline / Pascal Poirier / texte établi et annoté par Judith Perron, Moncton (N.-B.), Éd. d’Acadie, 1998, 128 p.

3. Alexandre Braud (1872-1939), membre de la communauté des pères eudistes (Congrégation de Jésus et Marie), vécut et travailla, toute sa vie adulte, en Acadie 
au Collège Sainte-Anne (Pointe-de-l'Église, N.-É.). Cette pièce connut un succès immédiat, avec des commentaires élogieux dans les journaux locaux et surtout une critique très positive de l'éminent homme politique et écrivain nationaliste, Pascal Poirier, intitulée «Un poème acadien »" Le titre de l'article doit retenir notre attention, car l'épithète est capitale : elle constitue une reconnaissance de l'appartenance de l'œuvre au patrimoine littéraire acadien de la part d'une des figures de proue de la Renaissance acadienne. La pièce interpelle la collectivité : " C'est un chant au patriotisme et à la loyauté. [...] Les personnages : Subercase, Morpain, SaintCastin, Le Borgne, De La Ronde, La Tour, Desgoutins, Gaudet, Belliveau, Melanson, Thériault, et une fraîche et gracieuse figure d'enfant, Joanno, sont bien ce qu'il y a de plus acadien, dans notre histoire. ${ }^{5}$ P. Poirier intervint pour faire publier la pièce dans Le Moniteur acadien, du 14 août au 30 octobre $1902^{6}$.Celle-ci décrit et interprète plusieurs épisodes d'un événement clé de l'histoire de la collectivité acadienne en rapport avec ses origines, événement catastrophe qui retentit sur son identité et sur sa définition. En effet, la capitulation du gouverneur de Port-Royal, Daniel Auger de Subercase, en 1710, modifie définitivement le cours de l'histoire de cette population en y introduisant une rupture, la cession de son territoire. A. Braud représente les derniers jours de l'Acadie française.

La suite de la genèse de l'œuvre nous est connue grâce à un court texte de l'auteur intitulé "L'Origine de la pièce », publié en 19367. Il y

et au Québec. Pendant trente ans, il exerça la fonction de professeur dans plusieurs établissements d'éducation et celle de missionnaire à Rogersville (N.-B.) et à l'île d'Anticosti, sur la Côte-Nord (QC). Il fut aussi vicaire de plusieurs paroisses, dont celle du Saint-Cœur-de-Marie, à Québec, et aumônier attaché à des communautés religieuses et à d'autres groupes. Il eut une production littéraire diversifiée et substantielle : pièces de théâtre, poésies de circonstances et d'inspiration religieuse, articles religieux et historiques, communiqués d'intérêt régional et correspondance variée. C'est grâce aux journaux locaux, L'Évangéline et Le Moniteur acadien, que la plupart des travaux de l'auteur nous sont parvenus. Voir : René LeBlanc et Micheline Laliberté, Sainte-Anne, Collège et Université, 1890-1990, Pointe-de-l’Église, Université Sainte-Anne, 1990, 502 p.; Robert Viau, "Le Théâtre à Pointe-de-l'Église : Subercase ou les dernières années de la domination française en Acadie », dans Édouard Langille et Glenn Moulaison (dir.), Les abeilles pillotent, Mélanges offerts à René LeBlanc, Revue de l'Université Sainte-Anne, p. 119-141.

4. Pascal Poirier, "Un poème acadien », Le Moniteur acadien, 7 août 1902, p. 2, col. 3-4.

5. Ibid.

6. Alexandre Braud, "Subercase », Le Moniteur acadien, 14 août 1902, p. 1, col. 1-4; p. 7, col. 2-4; 21 août 1902, p. 1, col. 2-4; 25 septembre 1902, p. 1, col. 2-4; 2 octobre 1902 , p. 1, col. 2-4; 9 octobre 1902, p. 1, col. 2-4; 16 octobre 1902, p. 1 , col. 2-4; 23 octobre 1902, p. 1, col. 2-4; 30 octobre 1902, p. 1, col. 2-4.

7. Alexandre Braud, "L'Origine de la pièce » dans [Anonyme], "Séance dramatique » [Programme], Saint-Cœur-de-Marie (Québec), [1936], 8 f., CÉA Anselme-Chiasson, 
mentionne la représentation du second acte de la pièce, en 1912, au collège de Caraquet, ce qui confirme l'intérêt qu'elle suscitait encore. II y décrit aussi la perte du seul manuscrit qu'il détenait, dans l'incendie de cet établissement, en décembre 1915. Celle-ci devint très lourde quand il entreprit, en 1936, de reconstituer le texte original. Il séjournait à Québec depuis de nombreuses années quand un prêtre membre de la section paroissiale de la Société Saint-Jean-Baptiste dont il était aumônier lui demanda de représenter la pièce de nouveau. N'ayant plus de texte disponible, il se mit en rapport avec le fils de l'éditeur du journal qui avait publié plus tôt le feuilleton. Il en obtint seulement les deux premiers actes, si bien qu'il dut soumettre sa pièce à une réfection importante : "Mais le troisième acte manquait. Je décidai donc une refonte de la pièce, et c'est Subercase légèrement modifié dans les deux premiers actes, complètement transformé dans le troisième acte et dans l'épilogue, qui va être représenté aujourd'hui. " ${ }^{8}$

Ainsi, à 34 ans de distance, fut partiellement réécrite et transformée la pièce, sous une forme et avec un sous-titre différents : Subercase ou les Dernières années de la domination française en Acadie - Drame historique en trois actes et un épilogue. Elle fut jouée les 16 et 17 avril 1936 à la salle paroissiale du Saint-Cœur de Marie (Québec).

Sous quelle(s) forme(s) se présente le texte? Quelle place cette pièce occupe-t-elle dans le patrimoine théâtral acadien? Où l'auteur a-t-il puisé son inspiration? Peut-on rapprocher ce drame d'autres œuvres marquantes en Acadie et ailleurs au Québec et au Canada français? Telles sont quelques-unes des questions que nous pouvons nous poser aujourd'hui.

\section{Le texte}

À l'examen de l'édition de 1936, on se rend compte que l'on a affaire à un état du texte bien distinct. Ce dernier est, en réalité, constitué de deux sous-états, représentés par deux tapuscrits du texte de la pièce, très voisins, non datés, mais qu'on peut situer autour de la date des deux représentations (vers 1936). Le premier tapuscrit ${ }^{9}$ comprend 38 feuilles dactylographiées portant des corrections manuscrites de l'auteur. Le deuxième tapuscrit ${ }^{10}$ a une autre forme : une nouvelle frappe reproduit le

fonds Ferdinand-J.-Robidoux, 4.6-14.

8. Id., f. 6-7.

9. Alexandre Braud, Subercase ou les Dernières Années de la domination française en Acadie (Drame historique en trois actes et un épilogue, [tapuscrit avec corrections manuscrites de l'auteur à l'encre noire comprenant 4 feuillets agrafés], [1936], $13+14+7+4$ f, CÉA Anselme-Chiasson : fonds 529.1-1.

10. Alexandre Braud, Subercase ou les Dernières Années de la domination française en Acadie (Drame historique en trois actes et un épilogue), [tapuscrit relié à couverture bleue avec corrections manuscrites de l'auteur], [1936], CÉA Anselme-Chiasson, 
texte du tapuscrit précédent avec les corrections de l'auteur intégrées et de nouveaux écarts. Il lui est donc postérieur. C'est ce dernier état qui fait autorité, car il est l'expression de la dernière volonté de l'auteur; c'est celui que nous avons choisi comme texte de base.

L'étude des écarts entre l'édition journalistique et le tapuscrit retenu a permis de formuler une série d'observations ${ }^{11}$. La première édition présente des paratextes, deux dédicaces et un poème, pièces que le tapuscrit ne renferme pas. On constate que les écarts entre les deux textes sont peu fréquents dans le premier acte et plus nombreux dans le deuxième. Apparaissent, dans le tapuscrit, pour ce qui est de ces deux actes, des substitutions de mots ou d'expressions, quelques ajouts de vers pour la rime, des didascalies. Se remarquent aussi des condensations et des coupes de longues tirades, qui atténuent l'aspect déclamatoire et un certain statisme, caractéristiques de l'édition journalistique. Grâce aux échanges, ces modifications créent, sinon du mouvement, du moins une illusion de mouvement. Les formes originales corrigées par l'auteur constituent les variantes notées dans l'apparat critique de l'édition en cours de réalisation.

Quant à la suite de la pièce, on a affaire à une véritable réécriture. Tandis que cette division ne comprend qu'un acte, contenant lui-même quatre scènes, dans l'édition journalistique, elle renferme, dans le tapuscrit, un acte et un épilogue contenant chacun sept et deux scènes respectivement. La distribution des personnages subit des remaniements : il y a substitution d'enfant; le rôle de confident passe du corsaire Morpain à un nouveau personnage, le père Beaudoin. À Subercase, le gouverneur qui garde son honneur dans la défaite, qui transforme celle-ci en victoire, se substitue un nouvel héros, le prêtre, sur qui repose l'avenir d'un territoire qui fut la Nouvelle-France. Dans le tapuscrit apparaissent deux pièces finales, un chant et une dédicace. Comme le texte original du troisième acte ne se laisse pas réduire à l'état de variante, il est présenté intégralement, dans l'édition critique de l'œuvre, à la suite du texte du tapuscrit qui constitue le texte de base. En conséquence, seuls figurent dans l'apparat critique les mots et vers du premier tapuscrit corrigés par l'auteur.

Aucune autre édition n'est venue s'ajouter à celle de 1902. Le texte remanié de 1936 resta inédit. L'œuvre dramatique tomba dans un oubli qui dura plusieurs décennies. Ce ne fut qu'à la fin des années 1970 que l'on redécouvrit l'écrivain et que l'on étudia son rôle dans l'histoire du théâtre acadien. Dans l'anthologie qu'elle a codirigée, Marguerite Maillet cite deux

fonds A 842 B 62 s.

11. Pierre Gérin, «Un cas d’inachèvement, Subercase, œuvre littéraire acadienne stratifiée », dans Janine Gallant, Hélène Destrempes et Jean Morency, L'CEuvre et ses inachèvements, Longueuil (Qc), Groupéditions, 2007, p. 193-206. 
extraits du drame, d'après l'édition de 1902 : «Un conseil de guerre » (acte II, scène 2) et "Reddition » (acte II, scène 4) ${ }^{12}$. Elle présente aussi l'écrivain et son œuvre dans son Histoire de la littérature acadienne ${ }^{13}$. Quant à lui, Jean-Claude Marcus accorde au dramaturge plusieurs pages dans son étude sur "Les Fondements d'une tradition théâtrale en Acadie ${ }^{14}$. La pièce est décrite et commentée dans deux thèses portant sur le théâtre acadien ${ }^{15}$. Plus récemment, $A$. Braud a fait l'objet d'un article de Robert Viau, qui exploite une documentation riche et variée ${ }^{16}$.

\section{Le mythe}

Comment l'auteur s'est-il intéressé à la légende de Subercase et comment l'a-t-il adaptée? Dans son article sur la genèse de la pièce, il reconnaît avoir visité le site historique de Port-Royal : " [En visite à Annapolis] Mon temps étant très limité, une fois mon ministère dominical accompli, je me rendis au fort, l'examinai dans tous ses détails et je m'assis face à la mer, l'esprit et le cœur remplis de l'histoire de l'ancienne Acadie, insuffisamment connue d'un bon nombre. ${ }^{17}$ La reconstitution du passé conduit à l'écriture dramatique, qui, dans ce cas, exerce une fonction didactique, car elle assure la transmission de données historiques exemplaires à la communauté acadienne :

En méditant ces graves et grands souvenirs trop oubliés, je me disais qu'il serait peut-être utile de les faire revivre, dans une suite de tableaux [...] sur la modeste scène du collège. Mon ambition n'allait pas plus loin et je n'avais certes aucune prétention d'auteur dramatique en y songeant.

C'est de cette idée qu'est sorti «mon humble Subercase ». Je me proposais uniquement de rappeler à nos élèves et à nos paroissiens de la Pointe-de-l’Église un fait historique qui

12. Marguerite Maillet, Gérard LeBlanc et Bernard Émont, Anthologie de textes littéraires acadiens, Moncton, Éd. d'Acadie, 1979, p. 314-317.

13. Marguerite Maillet, Histoire de la littérature acadienne : de rêve en rêve, Moncton, Éd. d'Acadie, 1983, p. 134-137.

14. Jean-Claude Marcus, "Les Fondements d'une tradition théâtrale », dans Jean Daigle (dir.), Les Acadiens des Maritimes, Moncton, CÉA Anselme-Chiasson, 1980, p. 645, 656-658.

15. Roger Lacerte, « Le Théâtre acadien : Étude des principaux dramaturges et de leurs œuvres (1957-1977) ", thèse de doctorat, Boston College, 1984, f. 57-60; Judith Perron, "Théâtre, fêtes et célébrations en Acadie (1880-1980) ", thèse de doctorat, U. de Moncton, Faculté des arts, 1995, f. 76-84.

16. Viau, loc. cit.

17. Braud, art. cit., f. 3 . 
s'était accompli presque chez eux et dont ils avaient raison d'être très fiers. ${ }^{18}$

Il est permis, cependant, de penser que l'intérêt de l'écrivain pour ce haut fait et pour son auteur a été entretenu par la lecture d'un volume de l'historien français Edme Rameau de Saint-Père, Une colonie féodale en Amérique ${ }^{19}$, publié en 1877 , à Paris, qui devait se trouver dans la bibliothèque des religieux du Collège Sainte-Anne.

Cet érudit s'intéresse beaucoup à l'Acadie et au Canada français ${ }^{20}$; il a une correspondance suivie avec P. Poirier. Tout en conservant l'image romantique du héros abandonné de tous, transmise par l'histoire, il trace un portrait positif du gouverneur, qu'il présente comme un homme d'action réfléchi et énergique. Il exploite aussi le thème de la résistance héroïque et de la bravoure. Toutefois, c'est par une opération très littéraire, la transfiguration de l'histoire, que son récit se distingue. En effet, dans son texte, la défaite devient une victoire symbolique. Le héros " rusé » (on pense à Ulysse) obtient les honneurs de la guerre et des conditions avantageuses. On aboutit à un renversement de situation grâce à la vente à bon prix de l'artillerie. On a affaire à une interprétation héroïque de la légende originale. Il y a métamorphose.

Braud reprend les points essentiels du texte de Rameau. Le lecteur est sensible à la caractérisation positive de Subercase, dont les qualités sont bien mises en évidence, et à la victoire symbolique, les assiégés recevant les honneurs de la guerre. Il y a agrandissement du protagoniste et des autres personnages. Cependant, l'auteur dramatique se distingue, à son tour, de sa source par un travestissement de l'histoire. Comme l'a remarqué avec beaucoup de justesse Judith Perron, " la distribution de la bataille dramatisée de 1710 ressemble davantage à celle, historique, de l'assaut de 1707 duquel Subercase est sorti vainqueur ${ }^{21}$. En effet, parmi les actants majeurs de 1710, ni Morpain le corsaire, ni Bernard de SaintCastin, qui ne fut jamais officiellement chef des Amérindiens comme son père, ni Matthieu Desgoutins ne participèrent à l'affrontement final, non plus que Le Borgne, qui mourut en 1693. Dans la pièce, en revanche, ils servent d'adjuvants et font ressortir la forte personnalité du gouverneur. Il faut mentionner une entorse avec la réalité historique : Charles de SaintÉtienne de La Tour (né en 1593), fils du traître Claude de Saint-Étienne

18. Id., f. 3-6.

19. Edme Rameau de Saint-Père, Une colonie féodale en Amérique (L'Acadie, 16041710) par M. Rameau, Paris, Didier, 1877, p. 340-352.

20. Voir : Robert Pichette, Napoléon III, l'Acadie et le Canada français, Moncton (N.-B.), Éd. d'Acadie, 1998, p. 79-152.

21. Perron, op. cit., f. 79. 
de La Tour (vers 1570-après 1636), ne mourut pas au cours du siège de 1710 , mais en 1666. De son mariage avec Jeanne Motin, veuve de son rival Charles de Menou d'Aulnay, il eut cinq enfants, dont un fils qui reçut le même prénom que le sien (entre 1663 et 1668-1731). L'écart entre les dates permet de penser qu'il y a eu confusion ou glissement, dans l'esprit de l'auteur, entre les deux homonymes ${ }^{22}$. Une autre innovation de l'auteur dramatique apparaît dans la passation des pouvoirs entre le gouverneur et le père Beaudoin : elle assure la transition et explique la capitulation et sa conséquence, l'abandon. La foi catholique est la gardienne de la culture française en terre d'Amérique, la religion assure le lien entre le passé et le présent.

Par le truchement d'A. Braud, la légende de Subercase évolue : elle permet de substituer à une rupture une continuité; elle offre une justification compréhensible, admissible pour la collectivité. La cession de Port-Royal et conséquemment l'abandon des Acadiens s'expliquent par le destin du héros, qui lui fut contraire, ce qui permet de le grandir face à l'adversité et de faire admettre la réalité des faits : "L'histoire et l'avenir diront de vous Seigneur / Que Subercase fut plus grand que le malheur. „ ${ }^{23}$ Il y a une transformation, une métamorphose de l'histoire. La défaite est transformée en victoire symbolique. On passe de l'histoire au mythe et à sa représentation théâtrale. Certaines opérations sont clairement identifiables. Il y a agrandissement du héros et de son combat : le gouverneur est le défenseur de l'Acadie, de la France, du Roi et de la Croix; le conflit dépasse l'enjeu local. On assiste aussi à une transfiguration du conflit impérialiste, avec une exaltation de la mystique patriotique. Le mythe, cependant, évolue : le gouverneur cède son autorité au clergé catholique; le pouvoir religieux remplace alors le pouvoir politique. On arrive à une " conception religieuse du mythe ».

Ainsi, le mythe de Subercase répond bien aux trois fonctions du mythe distinguées par Pierre Brunel : "Le mythe raconte. Le mythe est un récit. [...] Le mythe explique. C'est la deuxième fonction. [...] Troisième fonction : le mythe révèle. ${ }^{24}$ En outre, tout historique qu'il est, il faut noter que le mythe de Subercase est littéraire du moment même qu'il est écrit, mais aussi que l'œuvre littéraire transforme à son tour le mythe.

22. Voir : George MacBeath, "Saint-Étienne de La Tour, Claude De », "Saint-Étienne de La Tour, Charles De (mort en 1731) ", "Saint-Étienne de La Tour, Charles De (1593-1666) ", " Motin (Mottin), Jeanne (Menou d'Aulnay; Saint-Étienne de La Tour »); voir aussi : René Baudry, "Menou d’Aulnay, Charles de », dans John English et Réal Bélanger (dir.), Dictionnaire biographique du Canada en ligne, 2003, 2008. http://www.biographi.ca (pages consultées le 15 novembre 2010)

23. Alexandre Braud, Subercase ou les Dernières Années..., III, 4, v. 1147-1148.

24. Pierre Brunel, «Préface », Dictionnaire des mythes littéraires, Paris, éd. du Rocher, 2000, p. 8-9. 


\section{Le théâtre collégial}

Écrit pour la scène d'un collège acadien, le drame Subercase traite un sujet et exploite des thèmes qui ne sont pas étrangers aux pièces produites ailleurs au Québec et au Canada français. Quelles qu'aient été les communautés religieuses qui assuraient alors l'enseignement et les provinces dans lesquelles elles exerçaient, les productions théâtrales des collèges présentent des caractéristiques communes.

On reconnaît la valeur formative du théâtre et on partage certains objectifs pédagogiques : outre l'histoire, l'accent est mis sur l'éloquence, le développement de la culture et la mémorisation de textes. On donne l'exemple du bien-dire. Ainsi que l'observe Jeanne Corriveau, "l'art dramatique fut dans nos institutions collégiales un instrument de culture largement utilisé pour la formation humaniste des élèves $»^{25}$. Les pères eudistes, qui dirigeaient un réseau d'établissements d'éducation au Canada, et plus particulièrement le Collège Sainte-Anne en NouvelleÉcosse, où enseignait $A$. Braud, valorisaient, eux aussi, le théâtre. À ce sujet, R. Viau fait l'observation suivante : "Dès 1893, des pièces étaient présentées au collège Sainte-Anne [...]. Avant 1920, on ne montait pas plus de deux pièces par année au Collège mais en 1920, 1925, 1928 et 1932, le nombre passe à quatre et, en 1927, à cinq pièces. " ${ }^{26}$ Il faut préciser que, dans les collèges canadiens-français, on ne se limite pas au seul genre dramatique : "Tous les genres, tragique, comique ou dramatique se partagent apparemment sans suite ou influence repérable les sujets religieux, historiques ou patriotiques les plus divers. ${ }^{27}$

Quant au contexte de la représentation, il faut mentionner que les pièces étaient jouées lors de manifestations appelées séances. J.-C. Marcus s'est intéressé à ces dernières en Acadie :

À partir de la fin des années 1870 et pendant plus d'un demisiècle, on assiste ainsi dans tous les coins de l'Acadie [...] à une prolifération des séances. Il serait presque illusoire de vouloir en dresser un répertoire tant leur nombre est considérable. S'il n'est guère contestable que les premières d'entre elles eurent lieu dans les collèges et couvents [...], il ne faudrait surtout pas croire qu'elles ont été l'apanage des seules maisons d'enseignement. Car [...] les séances, données

25. Jeanne Corriveau, "Jonathas du R. P. Gustave Lamarche et le théâtre collégial ", Montréal, Université de Montréal, 1965, f. 9 [mémoire de maîtrise].

26. Robert Viau, "Jean-Baptiste Jégo, pionnier du théâtre acadien », Revue de l'Université Sainte-Anne, 1996, p. 116.

27. Corriveau, op. cit., p. 30. 
dans les paroisses, participent pleinement de la vie sociale des Acadiens [...]. ${ }^{28}$

Or la pièce d'A. Braud a été jouée dans le cadre de séances dramatiques qui relèvent du théâtre collégial et du théâtre paroissial. Dans le cas de la vie collégiale, le théâtre est au programme des cérémonies qui ont lieu à l'occasion de la visite d'un dignitaire. C'est ainsi que la première représentation de Subercase s'intègre à la "Séance Dramatique, Littéraire et Musicale, offerte Au Très Rév. Père Dagneau, supérieur, à tous les Pères et amis du Collège Ste-Anne, par les membres du "Cercle Littéraire St Jean l'Évangéliste", en témoignage de notre reconnaissance ${ }^{29}$. Quant à celles des 16 et 17 avril 1936, elles sont placées sous une double autorité religieuse et politique : "La représentation de ce soir sera sous la présidence d'honneur du T. Rév. Père Frs Tressel, provincial des Eudistes, et celle de demain soir sous celle de M. J.-Bona Arsenault, président de la section locale de la Société acadienne L'Assomption. " ${ }^{30}$ C'est une série intéressante de descriptions de séances que livrent les journaux locaux, dans leurs critiques des spectacles : on dépeint la salle des fêtes, les décors, les banderoles, les costumes; on croque le public; on n'oublie pas les notables; on note chaque point du programme, sans omettre les allocutions et les hymnes. Ainsi, la pièce fait partie d'un ensemble plus vaste : elle est précédée et suivie d'autres œuvres artistiques (en Acadie, plusieurs en anglais). Elle est découpée, prise en sandwich. Mais le public est gagné d'avance, il est préparé, il attend le dénouement. On doit ajouter que, pour lui, les séances constituent, le plus souvent, la seule forme d'exposition au théâtre et une sorte d'initiation à l'art dramatique : «[...] pendant une bonne période de notre histoire, ce sont nos collèges et nos couvents qui, [...], perpétuèrent le goût de l'art dramatique comme instrument de culture et moyen de maintenir à la langue son prestige et sa pureté $»^{31}$.

Enfin, le principal élément que partage Subercase avec maintes productions du théâtre collégial acadien, québécois et canadien-français du début du $x x^{e}$ siècle pourrait bien être la didactique de l'histoire. C'est d'ailleurs le titre ainsi formulé, "Théâtre : Histoire sur la scène ${ }^{32}$ ", que donne M. Maillet à un chapitre de son histoire littéraire acadienne portant sur le théâtre. A. Braud reconnaît avoir eu cet objectif, auquel s'associent

28. Marcus, art. cit., p. 637.

29. Observateur, "Collège Ste-Anne, Church Point ", L'Évangéline, 24 avril 1902, p. 3, col. 3 .

30. [Anonyme], « Subercase ", L'Action catholique, jeudi 16 avril 1936, p. 12, col. 6.

31. Jean Béraud, 350 ans de théâtre au Canada français, Montréal, CLF, 1958, p. 17.

32. Maillet, op. cit., p. 133. 
des valeurs morales comme le courage, la persévérance, l'imitation des ancêtres et le patriotisme. C'est précisément l'exaltation de ces dernières qu'a retenue $P$. Poirier, dans sa critique de la pièce qu'il élève au rang de poème héroïque. Un peu plus tard que son prédécesseur, un autre dramaturge acadien, James E. Branch (1907-1980), confirme la richesse du fonds historique acadien : "[L]'histoire acadienne est un tissu de drames poignants, une source inépuisable où l'amateur de théâtre peut puiser à loisir des thèmes d'inspiration. „33

Une telle orientation historique fondée sur ces valeurs convenait bien aux tenants du nationalisme en pleine Renaissance acadienne (18641930). En effet, cette période se caractérise par une prise de conscience collective de la communauté acadienne et par l'affirmation de son existence. Les Acadiens commençaient à se donner des institutions : une ligue pour la défense des intérêts collectifs, la Société nationale l'Assomption, des collèges et des journaux. Une idéologie nationaliste, partagée par les leaders religieux et laïcs acadiens, se répandait : elle visait à regrouper les forces nationales dispersées, à donner à la population le sentiment d'appartenir à une collectivité, en lui faisant redécouvrir ses racines et en lui donnant les moyens d'assumer son destin. Elle a été bien formulée dans la devise du Moniteur acadien : "Notre langue, notre foi, nos coutumes ». Le nationalisme professé dans Subercase, comme celui de maints orateurs des conventions nationales, repose sur cette trinité idéologique. P. Poirier ne pouvait pas y être insensible. A. Braud reprend l'essentiel de ces fondements idéologiques dans la formulation des objectifs pédagogiques visés par les collèges des Maritimes où l'on enseigne en français :

Nous avons surtout l'ambition de leur inculquer dans l'âme [aux élèves] l'amour de l'Acadie, de sa foi, de son drapeau et de sa langue, [...]. / [...] Vous aussi demeurez invinciblement attachés aux gloires de votre passé, vous aussi gravez avec fidélité et amour vos traditions d'honneur, de patriotisme et de foi. ${ }^{34}$

La mythification d'un personnage historique avec une victoire symbolique n'est pas propre à cette pièce. On en retrouve plusieurs exemples à la fois dans le théâtre collégial et public québécois et canadien-français de la même époque. Les sujets héroïques sont souvent traités par plu-

33. James E. Branch, L'Émigrant acadien - Drame social acadien en 3 actes, [Moncton, L’Évangéline Itée, 1929], p. 4.

34. [Alexandre Braud], "Conférence du R. P. Braud ", Le Moniteur acadien, 19 septembre 1901, p. 3, col. 1. 
sieurs dramaturges, les pièces écrites en vers et en prose, et l'on a affaire à des quasi-cycles.

Une œuvre annonce le drame acadien, Le Jeune Latour (1844) d'Antoine Gérin-Lajoie ${ }^{35}$. À vrai dire, P. Poirier lui-même invite à faire ce rapprochement :

Subercase est la deuxième tragédie en vers et en trois actes que notre histoire inspire à la poésie : la première est Charles Latour [il désigne l'œuvre par le nom du protagoniste], du sympathique auteur de Un Canadien errant, feu Gérin-Lajoie. Il serait très intéressant de comparer entre eux les poëmes de ces deux chantres de deux grandes gloires acadiennes. ${ }^{36}$

Écrite par un collégien pour un théâtre collégial, celui du collège de Nicolet (Québec), elle fut jouée pour la première fois en 1844. Cette tragi-comédie a une thématique acadienne : la scène se passe en mai 1630, au Capde-Sable (appelé aussi le Cap-Sable), à l'extrémité sud de la NouvelleÉcosse. Elle exploite un sujet historique à travers le prisme déformant de la peinture des relations conflictuelles entre un père et son fils. Elle présente un abus d'autorité de la part du père, qui s'est avili par sa trahison, et la résistance inflexible du fils, qui reste fidèle à la mère patrie et qui refuse de livrer le fort à l'Angleterre. Pareille opposition ne se retrouve pas chez Braud, où le fils du traître occupe une place beaucoup moins importante : seul est noté son désir obsessif de faire disparaître la tache honteuse et de racheter l'honneur familial :

Je vais comme un forçat qui doit traîner sa chaîne.

Et semblable à Judas, je demeure flétri.

Un stigmate honteux sur mon front est écrit.

Ciel! quand pourrais-je enfin laver cette souillure

35. Antoine Gérin-Lajoie, Le Jeune Latour, Montréal, Réédition-Québec, 1969 (éd. orig. 1844).

36. Pascal Poirier, "Un poème acadien », Le Moniteur acadien, 7 août 1902, p. 2, col. 4. Si le gouverneur Subercase mérite l'appellation de " gloire acadienne », tel n'est pas le cas de Charles de Saint-Étienne de La Tour. Attentif à l'action d'éclat avérée et fidèle à l'interprétation de l'auteur, Pascal Poirier omet de considérer la suite de l'existence de cet homme, assez glauque. II ne dispose pas, à son sujet, des mêmes documents que Maurice Lemire qui tient sur la caractérisation du personnage par l'auteur un jugement tout autre: "On peut lui reprocher [à Gérin-Lajoie] d'avoir fait d'un bandit un modèle de bravoure et de fidélité; sa jeunesse et sa connaissance imparfaite de l'histoire l'excusent. " Maurice Lemire, "Le Jeune Latour, tragédie d’Antoine Gérin-Lajoie », Dictionnaire des œuvres littéraires du Québec, Montréal, Éditions Fides, 1980, t. 1. Accessible en ligne : http://services.banq.qc.ca/sdx/ DOLQ (page consultée le 15 novembre 2010). 
Faire que mes enfants puissent, sans flétrissure,

Dire qu'ils ont appris l'honneur à mes côtés?37

Quant à elle, la dernière bataille de la Nouvelle-France est transformée en victoire symbolique et le personnage historique, Montcalm, en héros, dans Montcalm et Lévis (1918) d'Adolphe-Basile Routhier ${ }^{38}$. Mais aucune métamorphose héroïque n'atteint le niveau de celle de Dollard des Ormeaux, au Québec. En effet, le Dictionnaire des œuvres littéraires du Québec a retenu six créations dramatiques distinctes (textes et représentations) entre 1911 et 1938 , qui le mettent en scène ${ }^{39}$.

$$
\text { * } \star *
$$

Exercice pédagogique qui promeut les fondements de l'idéologie nationaliste, par le truchement de la récupération du mythe d'un héros fondateur, le drame d'A. Braud est une œuvre à l'histoire singulière. L'édition critique en cours de réalisation est l'occasion de faire une nouvelle lecture de ce texte accessible uniquement dans un dépôt d'archives, de le publier intégralement avec des annotations et des commentaires, de rendre compte de sa genèse grâce à un apparat critique et de le situer dans ses multiples contextes.

Par-delà l'examen d'une œuvre spécifique, cette édition vise à faire connaître ou redécouvrir une forme d'art dramatique, certes tombée en désuétude, mais qui ressortit aux patrimoines culturels acadien, québécois et canadien-français. Celle-ci a eu son heure de gloire et de succès; elle est encore très proche de nous chronologiquement; elle a surtout eu le mérite d'avoir contribué à la défense de la langue et de la culture françaises au Québec et au Canada.

37. Alexandre Braud, Subercase..., II, 1, v. 539.

38. Adolphe-Basile Routhier, Montcalm et Lévis - Drame historique en cinq actes avec prologue et six tableaux, Québec, Imprimerie franciscaine missionnaire, 1918, $173 \mathrm{p}$.

39. Ce sont, respectivement : Olivier-Bourbeau-Victor Rainville, Dollard des OrmeauxDrame en vers en neuf tableaux, Montréal, Librairie Beauchemin limitée, [1911], 166 p.; Hervé Gagnier, Dollard - Pièce en trois actes et cinq tableaux, Montréal, Imprimerie des Éditeurs limitée, 1922, 79 p.; Julien Perrin, Gloire à Dollard - Pièce historique en cinq tableaux, Montréal, Bibliothèque de l'Action française, 1923, 34 p.; Émilien Gauthier, Dollard n'est pas mort! - Drame en deux actes tout spécialement écrit pour nos collégiens, Québec, l'Action sociale (limitée), 1927, 56 p.; Aldéodat Lavoie, Dollard (Sacrifice du Long-Sault) - Poème dramatique en cinq actes, Avignon, Maison Aubanel Père, 1937, 123 p.; Gire Maiguéret [Aimé Giguère], Dollard - Drame en trois actes (en vers), Montréal, [Imprimé au « Devoir »], 1938, $202 \mathrm{p}$. 